\title{
Preparation of a three-dimensional extracellular matrix by decellularization of rabbit livers
}

\author{
Gustavo A. Nari ${ }^{1,2}$, Mariana Cid ${ }^{3,4}$, Romina Comín ${ }^{3}$, Laura Reyna ${ }^{5}$, Gustavo Juri ${ }^{6}$, Ricardo Taborda ${ }^{5}$ \\ and Nancy A. Salvatierra ${ }^{3,4}$ \\ ${ }^{1}$ Department of Surgery. Florencio Díaz Hospital. Argentina. ${ }^{2}$ Surgery UHC 4. UNC. Argentina. ${ }^{3}$ Department of \\ Chemistry. School of Biomedical Engineering. Faculty of Exact, Physical and Natural Sciences. National University \\ of Córdoba. Argentina. ${ }^{4}$ IIBYT-CONICET. Argentina. ${ }^{5}$ LIADE (Laboratory of Applied Research, and Development). \\ School of Biomedical Engineering. Faculty of Exact, Physical and Natural Sciences. National University \\ of Córdoba. Argentina. ${ }^{6}$ School of Biomedical Engineering, Faculty of Medical Sciences. UNC. Argentina
}

\begin{abstract}
Introduction: the availability of transplantable livers is not sufficient to fulfill the current demand for grafts, with the search for therapeutic alternatives having generated different lines of research, one of which is the use of decellularized three-dimensional biological matrices and subsequent cell seeding to obtain a functional organ.

Objective: to produce a decellularization protocol from rabbit liver to generate a three-dimensional matrix.

Methods: a combination of physical, chemical (Triton X-100 and SDS) and enzymatic agents to decellularize rabbit livers was used. After $68 \mathrm{~h}$ of retrograde perfusion, a decellularized translucent matrix was generated. To evaluate if the decellularization protocol was successful, with the extracellular matrix being preserved, we carried out histological (light microscopy and scanning electron microscopy) and biochemical (DNA quantification) studies.

Results: the decellularization process was verified by macroscopic observation of the organ using macroscopic staining, which revealed a correct conservation of bile and vascular trees. A microscopic observation corroborated these macroscopic results, with the hematoxylin-eosin staining showing no cells or nuclear material and the presence of a portal triad. Wilde's staining demonstrated the conservation of reticulin fibers in the decellularized matrix. In addition, scanning electron microscopy revealed a preserved Glisson's capsule and a decellularized matrix, with the DNA quantification being less than $10 \%$ in the decellularized liver compared to control. Finally, the time taken to develop the decellularization protocol was less than 96 hours.

Conclusions: the proposed decellularization protocol was correct, and was verified by an absence of cells. The hepatic matrix had preserved vascular and bile ducts with a suitable three-dimensional architecture permitting further cell seeding.
\end{abstract}

Key words: Decellularized liver. Biological scaffolds. Transplant.

Received: $10-10-2012$

Accepted: 26-12-2012

Correspondence: Gustavo Adrián Nari. Department of Surgery. Hospital Florencio Díaz. Argentina

e-mail: gusnari@hotmail.com
Nari GA, Cid M, Comín R, Reyna L, Juri G, Taborda R, Salvatierra NA. Preparation of a three-dimensional extracellular matrix by decellularization of rabbit livers. Rev Esp Enferm Dig 2013; 105:138-143.

\section{INTRODUCTION}

Liver transplantation is currently the only therapeutic alternative for acute liver failure, terminal liver disease or metabolic disorders originating in the liver. However, due to a shortage of donors, many patients who are on the waiting list never receive liver transplantation and others with indication of the graft have no access to waiting lists. Moreover, the complexity of the function of the liver makes it impossible to use artificial systems to provide temporary hepatic support as for example in patients with renal insufficiency.

Alternatives to transplantation, such as the non-biological support systems proposed in the literature, have precise and limited indications $(1,2)$. In other way, hepatocyte seeding, via injection into the splenic or portal veins, does not even offer a temporary solution as a bridge to transplantation, due to early death of hepatocytes with the remaining number being incapable of correcting any abnormalities or rescuing patients from liver failure. In facts, hepatocyte primary cultures lose their typical morphologies and function within a few days via dedifferentiation or epithelial-mesenchymal transition. Furthermore, other complications inherent to this technique, such as portal thrombosis, hypertension and pulmonary embolism, have also been reported $(1,3,4)$.

Three-dimensional biological scaffolds are commonly used in reconstructive surgery. Vracko (5) describes the importance of the extracellular matrix (ECM) in maintaining the liver structure and regeneration and recent developments show the ability of the ECM to maintain the hepatocyte phenotype and function $(1,4,6)$. Related to this, another line of research is the creation of three-dimensional 


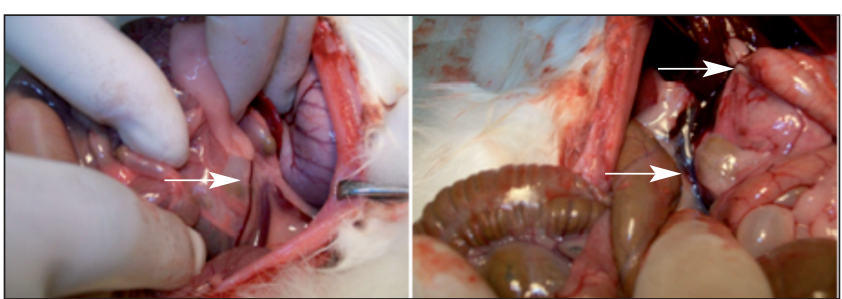

Fig. 1. A. Mesenteric vein (white arrow filled). B. Portal triad and inferior infra-hepatic caval vein (small white arrows).

scaffolds of ECM through a decellularization process of the liver, where hepatocytes, stem cells or other cells can be seeded to differentiate into hepatocytes in order to achieve a transplantable human liver. Researchers have proposed different models of decellularization to obtain a biological scaffold to allow subsequent cell seeding $(1,3,4,7-$ 11). The decellularization is obtained through the use of different physical and/or chemical mechanisms to break down organ cells and leave only the ECM, which then provides support to posterior cell seeding (12).

The aim of this paper is to provide a liver decellularization technique in rabbit liver and to evaluate the outcome of this method.

\section{MATERIAL AND METHODS}

For this study, six New Zealand male rabbits of 2,100 \pm $120 \mathrm{~g}$ were subjected to a total hepatectomy with preservation of the vasculature. One rabbit was assigned for a study of anatomy of the region, another being used for liver histological control and the quantification of the DNA. The livers of the four remaining rabbits were incorporated into the decellularization protocol. All procedures were conducted in accordance with the NIH Guide for the Care and Use of Laboratory Animals, as approved by the Animal Care and Use Committee of the Universidad Nacional de Córdoba, Argentina, and efforts were made to minimize animal suffering and the number of animals used.

\section{Surgical technique}

Anesthesia was performed by injecting with $35 \mathrm{mg} / \mathrm{kg}$ ketamine and $5 \mathrm{mg} / \mathrm{kg}$ xylazine intramuscularly (IM) as a single dose. The rabbits were placed in the supine position with their legs fixed.

Surgery: By median incision which was later extended to the right hemithorax. Identification was made of the mesenteric vein and an intravenous injection was carried out using 1,000 IU heparin.

Identification of the portal triad was performed and attached by a ligature, identification and dissection of the infra-hepatic inferior caval vein was carried out. The adhesions of the liver were removed and the incision was extending into the thorax.

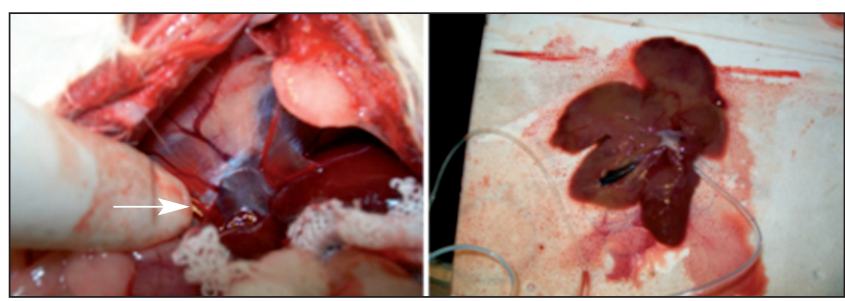

Fig. 2. A. Inferior supra-hepatic caval vein (white arrow). B. Total hepatectomy with catheter in perfusion stage prior to freezing.

Identification, dissection, ligature and cutting of the suprahepatic inferior caval vein were performed along with a total hepatectomy (Figs. 1 and 2)

Insertion of a catheter or teflon cannula in the suprahepatic or infra-hepatic inferior caval vein respectively was made, which was fixed with a prolene $4 / 0$ stitch and perfusion was started with PBS solution for washing.

The organ was placed in PBS solution with crushed ice for transport and subsequent freezing at $-80^{\circ} \mathrm{C}$.

\section{Decellularization protocol}

The liver was frozen in $500 \mathrm{ml} \mathrm{PBS}$ for $24 \mathrm{~h}$ at $-80{ }^{\circ} \mathrm{C}$ and later thawed at room temperature to aid cell lysis.

The catheter or teflon cannula inserted into the inferior caval vein was connected to a peristaltic pump and retrograde perfusion cycles was started at a rate of between 6 and $10 \mathrm{ml} / \mathrm{min}$ as follows: first, the liver was washed for 4 hours with $0.02 \%$ trypsin and $0.05 \%$ EDTA in deionized water, before being perfused with $3 \%$ Triton X-100 and $0.05 \%$ EGTA in PBS. Three washes of two hours each were performed, followed by a wash of 16 hours and later 3 washes of 2 hours each. Next, we used the ionic detergent SDS (0.1\% in deionized water), which was perfused for 4 hours, and then the organ was perfused with Triton X100 and EGTA (3\% and $0.05 \%$ in PBS) for 14 hours. Subsequent successive washes were performed with PBS and deionized water to remove cell remains and detergents (1 hour of deionized water, 2 washes with PBS for 1 hour, 30 minutes of deionized water, and two washes for $30 \mathrm{~min}$ with PBS). Finally, the organ was perfused for 1 hour with $4 \%$ ethanol in PBS to disinfect the organ.

\section{Microscopic evaluation}

- Light microscopy. For evaluation of the effectiveness of the decellularization, the two histological staining techniques, hematoxylin-eosin (HE), and the latter Wilde silver staining was used to evaluate reticular fibers (collagen type III). These stainings were performed on the decellularized and control livers.

- Scanning electron microscopy. A complete analysis of Glisson's capsule and the liver parenchyma was 


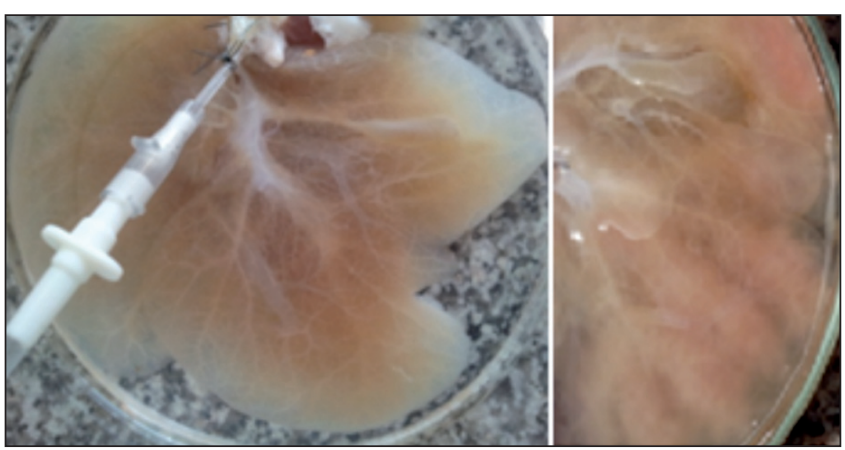

Fig. 3. Liver at different stages of process of decellularization. Note the appearance of the vasculature.

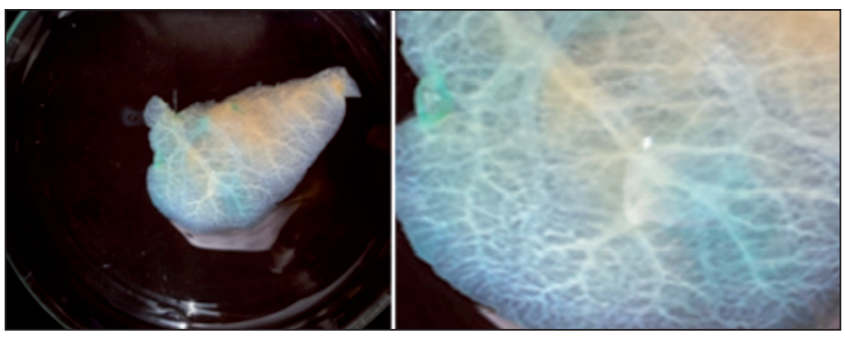

Fig. 4. A. Completely decellularized lobe. B. Zoom in. Vascular pattern preserved.

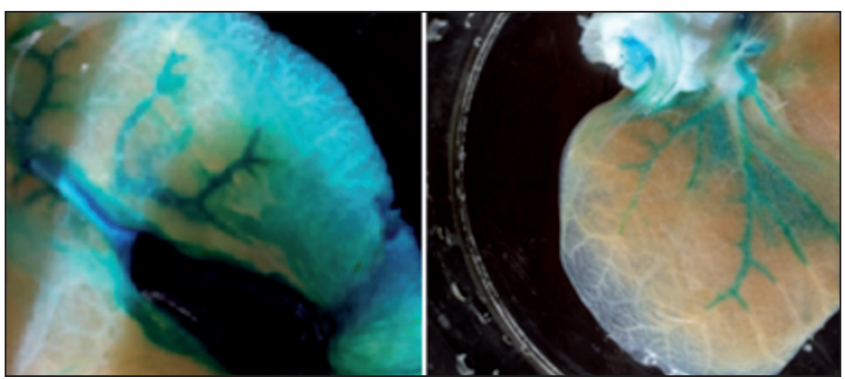

Fig. 5. A. Staining of bile ducts by vesicular puncture. B. Vascular staining by injection of methylene blue with agarose.

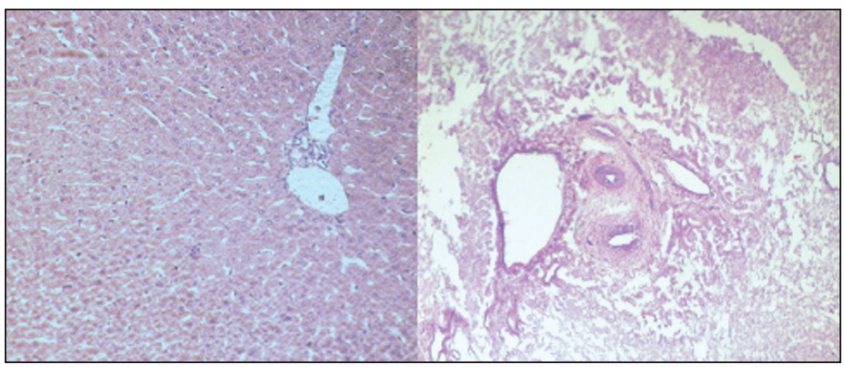

Fig. 6. Histological comparison of a normal liver (left) and a decellularized matrix (right). Hematoxylin-Eosin staining. Note the presence of portal triad in both preparations and the absence of cells and nuclei in the decellularized matrix (photos are representative of an experimental series, $n=4)$. performed by scanning electron microscopy (SEM) in LASEM, INIQUI-CONICET, UNSa using a scanning electron microscope model of JEOL JSM 6480 Brand LV.

\section{DNA quantification}

The amount of DNA was quantified using a method described by Laird et al. (13). Briefly, $25 \mathrm{mg}$ of tissue from the control and decellularized livers were homogenized in a solution containing $0.25 \%$ trypsin and $1 \mathrm{mM}$ EDTA in deionized water. The homogenate was incubated at $37{ }^{\circ} \mathrm{C}$ with constant stirring for 4 hours. Then, the cell lysis was continued with a solution containing $2 \%$ SDS, $5 \mathrm{mM}$ EDTA, $200 \mathrm{mM} \mathrm{NaCl}$ and $100 \mathrm{mM}$ TRIS-HCl, $\mathrm{pH} 8.5$ for $24 \mathrm{~h}$ at $55^{\circ} \mathrm{C}$. The DNA extraction was carried out in isopropanol and later dissolved in a solution of $10 \mathrm{mM}$ Tris$\mathrm{HCl}, 0.1 \mathrm{mM}$ EDTA, $\mathrm{pH} 7.5$. The amount of DNA was determined spectrophotometrically at $260 \mathrm{~nm}$.

\section{RESULTS}

The surgical technique was carried out without difficulties, and it was straight-forward to identify the anatomical elements. Cannulation of the inferior caval vein was performed twice in a supra-hepatic way and twice in an infrahepatic manner, the first with a K33 catheter and the second with a teflon cannula No. 16. The amount of PBS perfused for cleaning the liver prior to freezing was $210 \mathrm{ml}$.

The decellularization technique was performed as described previously on four rabbit livers and the total time taken was 94.30 hours, including 24 hours of freezing and 5 hours of thawing at room temperature (Figs. 3 and 4).

Staining of the biliary tree on two rabbits was performed with methylene blue through the gallbladder puncture, which revealed the existence of the bile ducts shown in Fig. 5. A mixture of methylene blue with agarose was perfused through the inferior caval vein in two other rabbits and a good vascular staining was observed (Fig. 5).

From the histological viewpoint, when the controls stained with $\mathrm{HE}$ and those with reticulin were compared using decellularized preparations, a complete disappearance of the cell pattern and the total absence of nuclei could be observed, with reticulin staining showing the presence of reticular fibers without cellular remains in the decellularized liver (Figs. 6 and 7, respectively). Furthermore, HE staining revealed the presence of a portal triad in the decellularized liver.

The SEM showed that the morphology of Glisson's capsule was similar to that of the liver control (Fig. 8), and revealed a complete decellularization of the liver parenchyma compared to control (Fig. 9). The Student's t-test for unpaired samples showed that the DNA quantification was significantly different $(t=4.151, \mathrm{p}<0.001)$ between the decellularized matrix values $(1958 \pm 579 \mathrm{ng} / \mathrm{mg}$ of tissue $)$ and the control ones $(192 \pm 53 \mathrm{ng} / \mathrm{mg}$ ) (Fig. 10). 


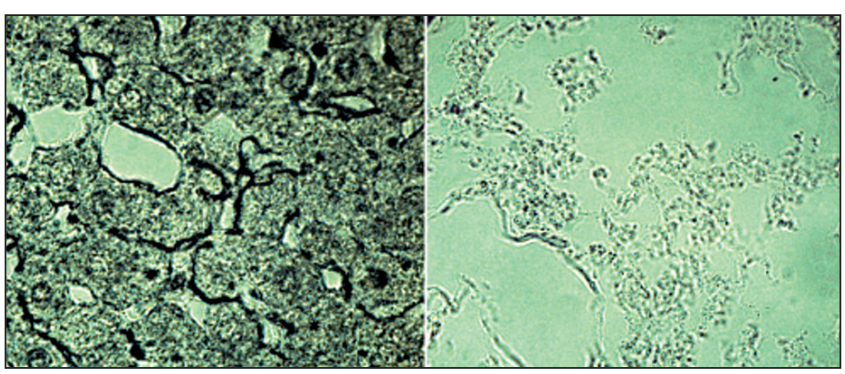

Fig. 7. Histological comparison of a normal liver (left) and decellularized matrix (right). Wilde's staining for reticulin (collagen III). On the right, note the absence of cells and presence of reticular fibers (the photos are representative of an experimental series, $n=4$ )

\section{DISCUSSION}

An insufficient availability of organs for transplantation has led to the search for alternative options to address this problem. Since the publication of successful heart decellularization by Ott et al. (14) and a posterior report on liver by Uygun et al. (8), several groups have begun to develop bioengineering techniques to obtain as a final aim a transplantable organ from a three-dimensional ECM scaffold, first involving organ decellularization, and later, the seeding of cell lines. The objective of the decellularization of the liver is to obtain a scaffold of ECM with a suitable architecture, contained in an intact Glisson's capsule and vascular and biliary structure preserved as much as possible $(4,8)$.

The techniques and materials used for this process are constantly being reviewed in order to achieve a greater cell lysis, which preserves the ECM and decreases the time required to carry it out. In the literature, different protocols have been modified and combined in different way, with
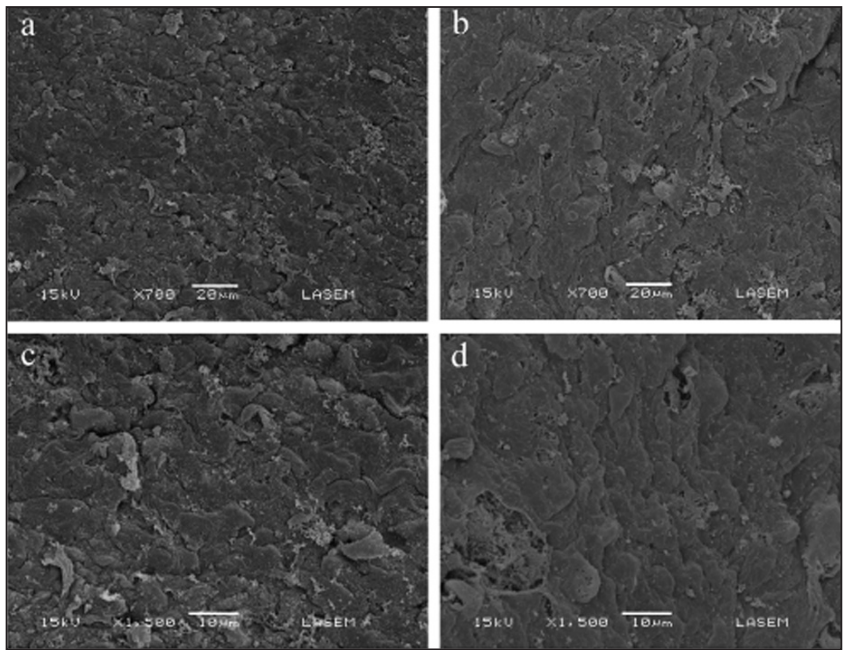

Fig. 8. Images of Scanning Electron Microscopy (SEM) of Glisson's capsule control ( $A$ and $C$ ), and decellularized liver capsule (B and D). Note the integrity of the capsule in the decellularized liver (photos are representative of an experimental series, $n=4$ ).

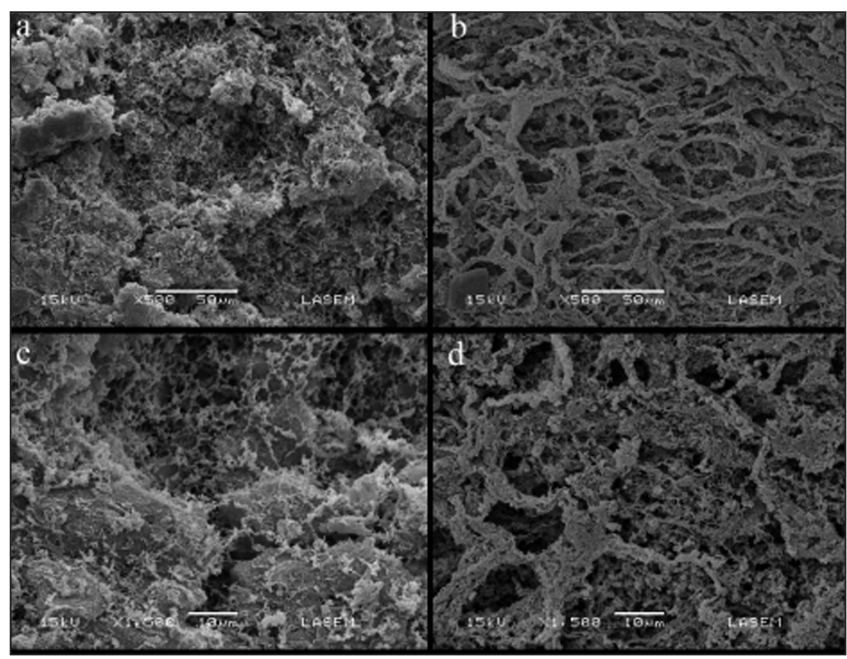

Fig. 9. Images of Scanning Electron Microscopy (SEM) of the control liver ( $A$ and $C$ ), and of the decellularized liver (B and D). Note the framework of the ECM without cells (photos are representative of an experimental series, $n=4)$.

respect to the physical and chemical agents used as well as the time of application $(12,15)$. The detergent is basically chosen by considering the characteristics of the organ or of the tissue to decellularize, e.g. decellularization of the tendon requires a different detergent than that for the lung or liver (12). To date, liver decellularization protocols have involved perfusion of a combination of detergents: Triton X-100 and SDS were used in the portal or caval vein, with a rat being the liver donor. However, Uygun et al. perfused $0.1 \%$ SDS (8), while Shupe and his colleagues used increasing concentrations of $0.1 \%$ Triton X-100 followed by SDS (11). Moreover, Soto-Gutierrez et al. first used an enzymatic agent: trypsin and then $3 \%$ Triton X-100 (4). When we previously tested these protocols $(4,11)$ a complete decellularization was not obtained. Therefore, we eliminated the perfusion with only SDS, as described in several reports because

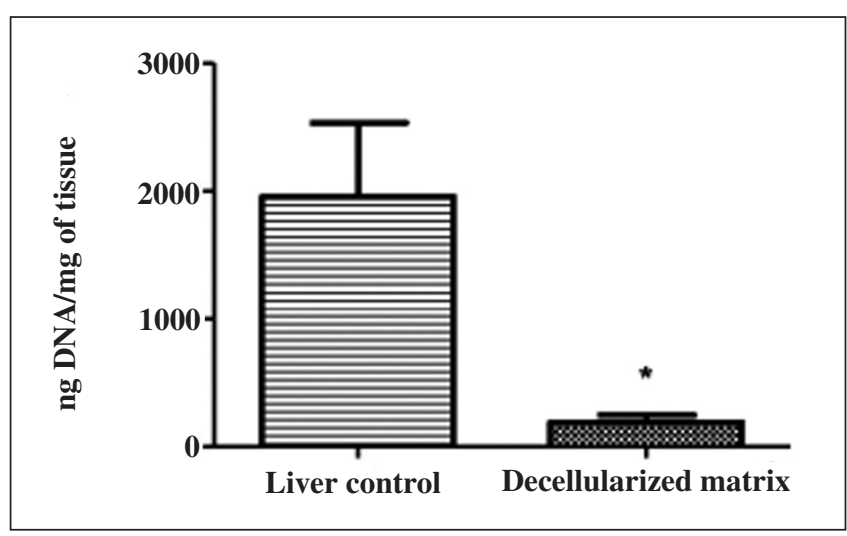

Fig. 10. Amount of DNA in control and decellularized livers. Bars represent mean \pm SEM. $\left({ }^{*} p<0.001\right.$ compared with liver control [Student's T-test] $[n=4])$. 
this technique results in a good decellularization by successfully removing cellular remains, but implied a higher loss of growth factors and of liver architecture (12) required for the process of recellularization.

The new protocol described in the present investigation involved an initial treatment with trypsin, which allowed a better transfer from the detergents to the tissue, then perfusion was carried out with $1 \%$ SDS and finally with Triton $\mathrm{X}-100$. Thus, the decellularization was performed using a combination of enzymatic treatment (trypsin), followed by an ionic detergent (SDS), and finally a nonionic one (Triton $\mathrm{X}-100)$. This strategy of using three different agents in the decellularization process minimized the contact time of each of these agents with the ECM, and also removed cells and their remains by three different mechanisms, thus faciliting a greater decellularization (12). Nevertheless, we wish to make it clear that changing the animal species implies a change in the size of the organ to be decellularized, which in turn means developing a new protocol as it complicates the entry of detergents into the tissue as well as the removal of cell remains, due to the increased size of the organ.

The evaluation of the results of decellularization of the livers is carried out by light microscopy (LM) with HE staining, scanning electron microscopy (SEM), and the presence of cell nuclei using the DAPI technique and/or DNA quantification by the determination of base pairs (bp). A successful decellularization is considered to have taken place when fragments are less than $300 \mathrm{bp}$ according to some authors, while others have proposed that these should be less than $200 \mathrm{bp}$. In this way, this would not generate any inflammatory response in the host $(4,16,17)$. The search for DNA traces in commercial scaffolds revealed cellular remains and DNA content, although these would not be able to trigger an inflammatory response. Moreover, the amount of Gal alpha 1,3 was also insufficient to trigger acute rejection, although it is known that Gal alpha 1,3 cause rejection in xenotransplantation $(16,18)$. Additionally, various studies, some of them on apoptotic porcine cells, have eliminated the possibility of the presence of DNA traces of retroviruses in biological scaffolds for xenotransplantation being able to transmit a disease to the host (19-21).

Our results have shown an encouraging decellularization by observing the HE staining in the light microscopy to corroborate the presence of a preserved ECM. Likewise, the staining for reticulin fibers (collagen type III) demonstrated their presence in the decellularized liver. We also observed the presence of a complete portal triad, an important fact as the preservation of bile ducts is a desired feature and is difficult to achieve in this process. The presence of a preserved bile tract enables posterior reconstruction with bile epithelial cells (4), in the same way that a preserved vascular network permits subsequent seeding with endothelial cells.

The microscopic visualization of the bile ducts was verified by macroscopic observation of the bile tree through the puncture and staining of the gallbladder. Furthermore, the vascular tree was preserved after the decellularization process, as evidenced by the perfusion of methylene blue in $2 \%$ agarose via the caval vein.

As noted here and in agreement with other authors, we consider that the use of combinations containing trypsin, SDS, Triton X-100 associated with EGTA, after freezing at $-80{ }^{\circ} \mathrm{C}$ is the best alternative to date to achieve an acceptable decellularization in a liver of about $100 \mathrm{~g}$, although the appearance of new products or the incorporation of other techniques, such as sonication or electroporation, may be included in the protocol in the future $(15,22)$. Most decellularized livers reported by other researchers came from rats, when involved decellularize sizes of up to 10 times lower than those of rabbits. Therefore, if a liver of the size of rabbit can be recellularized, it provides a transplantable cell mass with a subsequent better chance of recovery of the liver functions in patients (7). A surgical technique preserving the vascular and bile elements and Glisson's capsule as well as the insertion of the teflon cannula or catheter carefully permits a suitable perfusion in different lobes of the rabbit liver. In agreement with other authors, we performed a retrograde perfusion (caval-portal flow), due to it seeming to be better than anterograde perfusion (portalcaval flow), which was proposed by other researchers $(3,4)$. Similarly, a peristaltic pump with controlled flows ranging from 6 to $10 \mathrm{ml} / \mathrm{min}$ ensured a ECM with a conserved architecture. The decellularization protocol proposed here produced successful results with HE staining, a technique for collagen III, SEM and DNA quantification. In addition, the time required for decellularization, including freezing time, was less than 96 hours.

In conclusion, although the results are positive, there are still some points to be investigated. One of these is to verify the presence of growth factors in our ECM, because the posterior recellularization process requires their presence (23). These factors are essential to determine the behaviour, adhesion, migration, differentiation, proliferation and survival of seeded cells $(1,6,24)$. In a decellularization protocol with features similar to those described here, the remaining amount of growth factors was around 30-50\%, thereby allowing ECM recellularization with hepatocytes (4). Finally, and focusing on the recellularization, the efficient delivery of oxygen and nutrients to the culture is necessary in order to obtain a useful and functional organ.

\section{ACKNOWLEDGEMENT}

We thank Dr. Paul Hobson, native speaker, for the translation of the text and Silvia Blanco for the SEM technical assistance. N.A.S is a scientific career researcher of CONICET.

\section{REFERENCES}

1. Zhou P, Lessa N, Estrada D, Severson EB, Lingala S, Zern M, et al. Decellularized liver matrix as a carrier for the transplantation of human fetal and primary hepatocytes in Mice. Liver Transplantation 2011; 17:418-27. 
2. Rosa-Diez G, Gadano A. Sistemas no biológicos de soporte hepático artificial: ¿en qué consisten y que rol ocupan en la actualidad? Acta Gastroenterol Latinoam 2012;42:135-44.

3. Bao J, Shi Y, Sun H, Yin X, Yang R, Li L, et al. Construction of a portal implantable functional tissue-engineered liver using Perfusiondecellularized matrix and heptocytes in rats. Cell Transplantation 2011;20:753-66.

4. Soto-Gutierrez A, Zhang L, Medberry C, Fukumitsu K, Faulk D, Jiang $\mathrm{H}$, et al. A whole-Organ regenerative medicine approach for liver replacement. Tissue Engineering 2011;17:677-86.

5. Vracko R. Basal lamina Scaffold-anatomy and significance for maintenance of orderly tissue structure. Am J Pathol 1974;77:314-9.

6. Stellaro TR, Ranade A, Faulk DM, Mc Cabe GP, Dorko K, Badylak $\mathrm{SF}$, et al. Maintenance of human hepatocyte function in vitro by liver derived extracelular matrix gels. Tissue Eng 2010(Part A);16:1075.

7. Baptista P, Siddiqui M, Lozier G, Rodriguez S, Atala A, Soker S. The use of a whole organ decellularization for the generation of a vascularized liver organoid. Hepatology 2011;53:604-17.

8. Uygun BE, Soto-Gutierrez A, Yagi H, Izamis Ml, Guzzardi MA, Shulman C, et al. Organ reengineering through development of a transplantable recellularized liver graft using decellularized liver matrix . Nat Med 2010;16:814-20.

9. Badylak SF,Taylor D, Uygun K. Whole-organ tissue engineering: Decellularization and recellularization of three-dimensional matrix scaffolds. Annu Rev Biomed Eng 2011;13:27-53.

10. Park KM, Woo HM. Systemic decellularization for multi-organ scaffolds in rats. Transplant Proc 2012;44:1151-4.

11. Shupe T, Williams M, Brown A, Willenberg B, Petersen BE. Method for decellularization of intact rat liver. Organogenesis 2010;6: 134-36.

12. Crapo P, Gilbert T, Badylak S. An overview of tissue and whole organ decellularization processes. Biomaterials 2011;32:3233-43.

13. Laird P, Zijderveld A, Linders K, Rudnicki M jaenisch R et al. Simplified mammalian DNA isolation procedure. Nucleic Acids Research 1991;19:4293-5.
14. Ott H, Matthiesen T, Goh SK Black LD, Kren SM, Netoff T, et al. Perfusion-decellularized matrix: Using nature s platform to engineer a bioartificial heart. Nat Med 2008;14:213-21.

15. Azhim A, Yamagami K, Muramatsu K, Morimoto Y, Tanaka M. The use of sonication treatment to completely decellularize blood arteries: A pilot study. Conf Proc IEEE Eng Med Biol Soc 2011;2011:2468-71.

16. Gilbert TW, Freund J, Badylak SF. Quantification of DNA in biologic scaffold materials. J Surg Res 2009;152:135-9.

17. Nagata S, Hanayama R, Kawane K. Autoimmunity and the clearance of dead cells. Cell 2010;140:619-30.

18. Daly KA, Stewart-Akers AM, Hara H, Ezzelarab M, Long C, Cordero $\mathrm{K}$, et al. Effect of alphaGal epitope on the response to small intestinal submucosa extracellular matrix in a nonhumanun primate model. Tissue Eng Part A 2009;15:3877-88.

19. Bisset LR, Boni J, Lutz H, Schupbach J. Lack of evidence for PERV expression after apoptosis-mediated horizontal gene transfer between Porcine and Human cells. Xenotransplantation 2007;14:13-24.

20. Di Nicuolo G, Van der Kerkhove MP, Hoekstra R, Beld MG, Amoroso $\mathrm{P}, \mathrm{Battisti} \mathrm{S}$, et al. No evidence of in vitro and in vivo porcine endogenous retrovirus infection after plasmapheresis through the AMC-bioartificial liver. Xenotransplantation 2005;12:286-92.

21. Hermida-Prieto M, Domenech N, Moscoso I, Diaz T, Ishi J, Salomon DR, et al. Lack of cross-species transmission of porcine endogenous retrovirus (PERV) to transplant recipients and Abbatoir workers in contact with pigs. Transplantation 2007;84:548-50.

22. Sano MB, Neal RE, Garcia PA, Gerber D, Robertson J, Davalos RV, et al. Towards the creation of decellularized organ constructs using irreversible electroporation and active mechanical perfusion. Biomed Eng Online 2010;9:83-6.

23. Hammond J, Gilbert T, Howard D, Zaitoun A, Michalopoulus G, Shakesheff K, et al. Scaffolds containing growth factors and extracellular matrix induce hepatocyte proliferation and cell migration in normal and regenerative rat liver. J Hepatol 2011;54:279-87.

24. Burra P, Tomat S, Conconi MT, Macchi C, Russo FP, Parnigotto PP, et al. Acellular liver matrix improves the survival and functions of isolated rat hepatocytes cultured in vitro. Int J Mol Med 2004;14:511-5. 